\title{
One-Cycle Control of Switching Converters
}

\author{
Keyue M. Smedley, Member, IEEE, and Slobodan Ćuk, Senior Member, IEEE
}

\begin{abstract}
A new large-signal nonlinear control technique is proposed to control the duty-ratio $d$ of a switch such that in each cycle the average value of a switched variable of the switching converter is exactly equal to or proportional to the control reference in the steady-state or in a transient. One-Cycle Control rejects power source perturbations in one switching cycle; the average value of the switched variable follows the dynamic reference in one switching cycle; and the controller corrects switching errors in one switching cycle. There is no steady-state error nor dynamic error between the control reference and the average value of the switched variable. Experiments with a constant frequency buck converter have demonstrated the robustness of the control method and verified the theoretical predictions. This new control method is very general and applicable to all types of pulse-width-modulated, resonant-based, or soft-switched switching converters for either voltage or current control in continuous or discontinuous conduction mode. Furthermore, it can be used to control any physical variable or abstract signal that is in the form of a switched variable or can be converted to the form of a switched variable.
\end{abstract}

\section{INTRODUCTION}

$\mathbf{S}$ WITCHING converters are pulsed nonlinear dynamic systems. Such systems under proper pulsed nonlinear control should be more robust, have faster dynamic response, and provide better rejection of power-source perturbation than the same systems under linear feedback control. There has been a continuous effort in the power electronics community to search for large-signal nonlinear schemes to control switching converters.

In conventional feedback control, the duty-ratio is linearly modulated in a direction that reduces the error. When the power source voltage is perturbed, for example by a large step up, the duty-ratio control does not see the change instantaneously since the error signal must change first. Therefore a typical transient overshoot will be observed at the output voltage. The duration of the transient is dictated by the loopgain bandwidth. A large number of switching cycles is required before the steady-state is regained.

In current-mode control [3]-[5], a constant frequency clock turns the switch on at the beginning of each switching cycle. The switch current starts growing until it reaches the control reference, then the comparator changes its state and turns the transistor off. An artificial ramp is generally applied in order to eliminate the oscillation that occurs when the duty-ratio is greater than or equal to 0.5 . Theoretically, if the artificial ramp is chosen to be exactly equal to the falling slope $s_{f}$ of the inductor current, the system will reject the power source

Manuscript received September 22, 1993; revised June 13, 1995.

K. M. Smedley is with the Department of Electrical and Computer Engineering, University of California, Irvine, Irvine, CA 92717 USA

S. Cuk is with the California Institute of Technology, Pasadena, CA 91125 USA.

IEEE Log Number 9414900. perturbations in one cycle. This condition may be feasible in the case of a buck converter with a constant control reference. In general, the falling slope of the inductor current of a switching converter is a function of some dynamic states; therefore, it is not possible for the artificial ramp to match the falling slope of the inductor current in a transient. Due to this mismatch, current-mode control is unable to reject the power source perturbation in one switching cycle. In any case, if the control reference is a variable, current mode control is unable to follow the control reference or reject power source perturbations in one cycle no matter what artificial ramp is chosen and what type of converter is used.

In a feedforward buck converter, the power source voltage directly controls the duty-ratio before the output voltage error occurs. It may be able to isolate the output from the power source perturbation if the feedforward parameter is precisely designed and the switches are ideal. In reality, switches have turn-on and turn-off transients and an on-state voltage drop; hence, this scheme is not able to accurately reject the power source perturbation.

The ASDTIC converter introduced in [6] has a capacitor inverter that changes the unregulated power source voltage into a triangular waveform, "balanced AC waveform." The balanced AC waveform is rectified to form a train of unipolar triangles. A lowpass filter follows the rectifier to smooth the output waveform. The output voltage is controlled by adjusting the repetition rate of the triangle train. The control circuit includes an integrator that continuously integrates the error between the switched variable and the control reference in order to obtain zero average error in one switching cycle. In [7] an attempt was made to extend this control technique to control choppers at a constant switching frequency. In the steady-state, this continuous integration method guarantees that the average value of the switched variable equals the control reference. ${ }^{1}$ However, it takes many switching cycles to reach a new steady-state after a transient. This method is similar to the continuous-time linear integral control, which yields a zero steady-state error but a non-zero dynamic error. In addition, it is not stable when the duty-ratio is greater than or equal to 0.5 .

Sliding-mode control [8] is a nonlinear control method that defines sliding surface passing through a desired operating point. The trajectories of the two switch states reach the sliding surface from opposite sides and their velocity vectors have non-zero normal components in the vicinity of the sliding surface (reach condition). The switch flips after the motion reaches the hysteresis, $\Delta<\sigma<\Delta$, of the sliding surface such that the system motion is restricted along the sliding

\footnotetext{
${ }^{1}$ The average value of a switched variable is defined as the average in one switching cycle in this paper.
} 


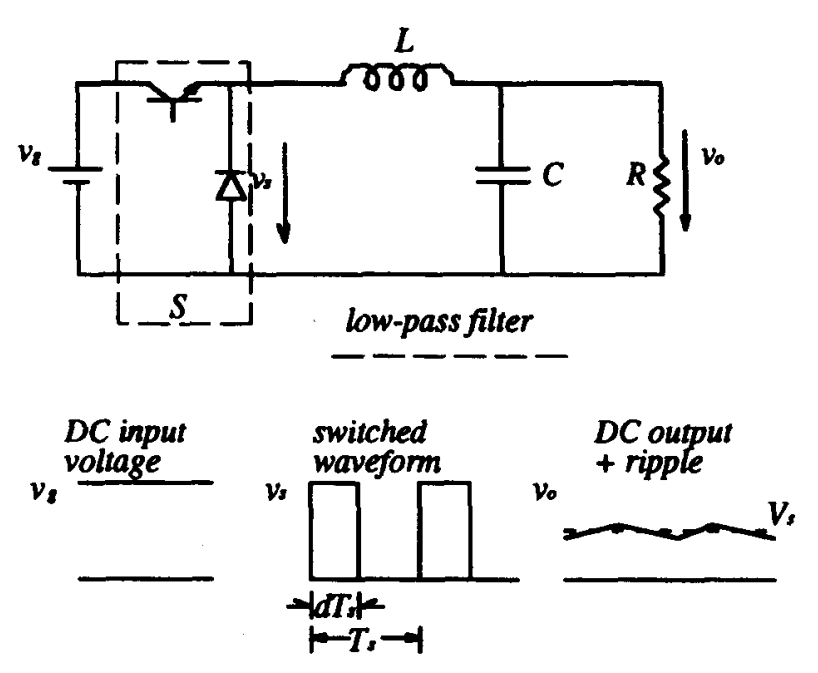

Fig. 1. The buck converter.

surface within the constant hysteresis. The system motion converges to the desired operating point if the average of the tangential components of the velocity vectors in every switching cycle points toward the operating point (converge condition). Usually, the motion converges to the operation point after many switching cycles; and the switching frequency is variable. If a variable hysteresis is used to envelope the system motion, the switching frequency can be fixed [9]. In general, a global sliding surface that satisfies both the reach condition and the converge condition may not exist.

A new nonlinear control technique, One-Cycle Control [1], [2], is introduced for constant switching frequency operation. This technique takes advantage of the pulsed and nonlinear nature of switching converters and achieves instantaneous dynamic control of the average value of a switched variable, e.g., voltage or current; more specifically it takes only one switching cycle for the average value of the switched variable to reach a new steady-state after a transient. There is no steadystate error nor dynamic error between the control reference and the average value of the switched variable. This technique provides fast dynamic response, excellent power source perturbation rejection, robust performance, and automatic switching error correction. This technique can be extended to control variable frequency switches. The One-Cycle Control technique is general and it is suitable for the control of pulse-widthmodulated (PWM) converters and resonant-based converters for either voltage or current control.

An application of a One-Cycle Controlled current-switch was reported in [10] based on [1] and [2]; however, the authors created a new name "Charge Control" disguising its true origin: One-Cycle Control.

Extensions and applications of One-Cycle Control reported in [11]-[14] have demonstrated the power of the One-Cycle Control method.

The One-Cycle Control theory is developed in Section II. Experimental results are provided that verify the feasibility of One-Cycle Control in Section III. The One-Cycle Control technique is generalized to control variable frequency switches, in Section IV. Conclusions and further discussions are given in Section V.

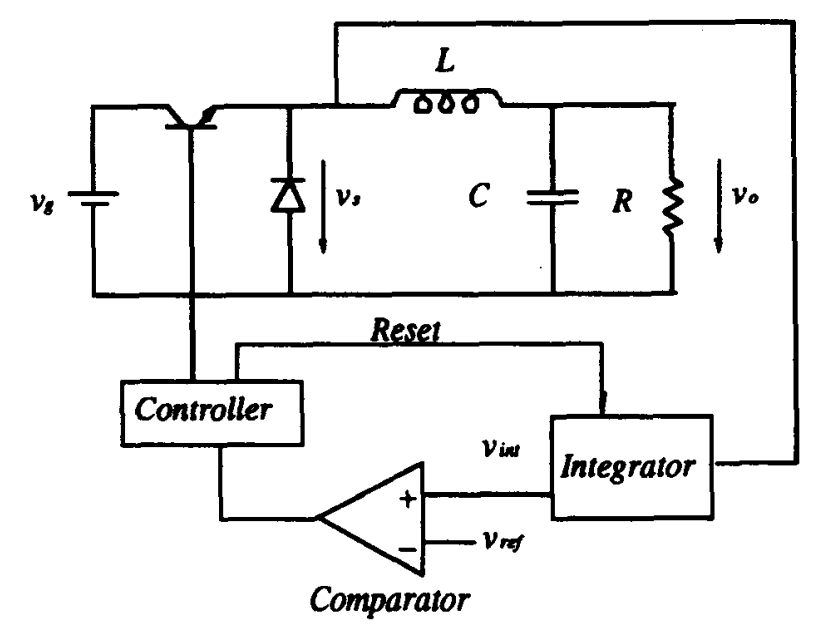

Fig. 2. One-cycle control of buck converter.

\section{ONE-CyCLE CONTROL}

The new control concept is presented on a buck converter as an example. This concept is generalized to control a switch for any form of signal, e.g. physical, electrical, mechanical, etc. Further analysis is given for the discontinuous conduction condition and for the automatic switching error correction feature.

\section{A. One-Cycle Control Concept}

A simple buck converter is shown in Fig. 1. The dc power source voltage is $v_{g}$ and the switch $S$ is operated with a constant frequency $f_{s}=1 / T_{s}$. When the transistor is on, the diode is off, and the diode-voltage $v_{s}$ equals the power source voltage $v_{g}$. When the transistor is off, the diode is on, and the diode-voltage $v_{s}$ is zero. The power source voltage is chopped by the switch resulting in a switched variable $v_{s}$. The LC lowpass filter transmits the average of the switched variable to the output while rejecting most of the undesirable switching frequency components. Therefore, the output voltage contains the desired DC value $d v_{g}$ and a small residual switching ripple.

Close observation of the switched variable leads to a simple fact. The output voltage of the buck converter is the average value of the switched variable, in this case the diode-voltage, that equals the area under each of the diode-voltage pulses divided by the switching period.

$$
V_{s}=\frac{1}{T_{s}} \int_{0}^{T_{s}} v_{s} d t=\frac{1}{T_{s}} \int_{0}^{d T_{s}} v_{g} d t .
$$

This observation provoked a new control scheme for constant switching frequency converters as shown in Fig. 2 . A constant frequency clock turns on the transistor at the beginning of each switching period. The diode-voltage is integrated and compared with a control reference. As soon as the integrated diode-voltage reaches the control reference, the comparator changes its state. As a result, the transistor is turned off and the integrator is reset to zero.

If the control reference is constant, then the average of the diode-voltage is constant and so is the output voltage as shown 


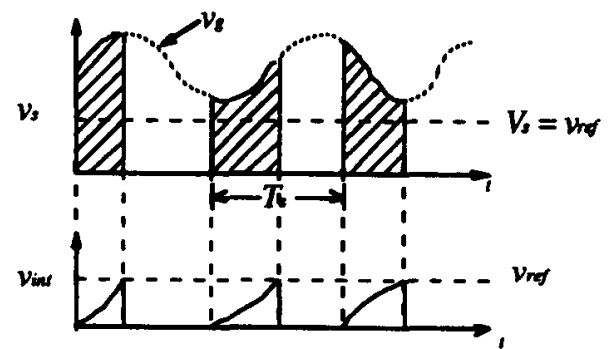

Fig. 3. Constant control reference.

in Fig. 3. The slope of the integration is directly proportional to the power source voltage. The integration value is continuously compared with the constant control reference. When the power source voltage is higher, the slope of the integration is steeper; therefore, the integration value reaches the control reference faster. As a result, the duty-ratio is smaller. When the power source voltage is lower, the duty-ratio is larger.

If the control reference is a function of time, then the average of the diode-voltage is equal to the time variant control reference in each cycle. Fig. 4 shows the case where the control reference changes its value with a single step up. The integration value of the diode-voltage keeps up with the control reference immediately.

With this control scheme, the duty-ratio $d$ is determined by

$$
\frac{1}{T_{s}} \int_{0}^{d T_{s}} v_{g} d t=v_{\text {ref }}
$$

The duty-ratio $d$ of the current switching cycle is independent of the state of previous switching cycles; therefore, the transient of the average value of the switched variable, the diode-voltage, is completed within one switching cycle. The name which most appropriately defines this new nonlinear control scheme is One-Cycle Control.

The duty-ratio governed by (2) is a nonlinear function of the power source voltage and the control reference. With this nonlinear control, the output voltage of the buck converter becomes a linear function of the control reference independent of the power source voltage,

$$
v_{o}=\frac{v_{\mathrm{ref}}}{1+\frac{L}{R} S+L C S^{2}} .
$$

In the case when there is an input filter in front of a buck converter, the control-to-output transfer function will have a maximum phase shift of $540^{\circ}$ due to the existence of the righthalf-plane zeros, which makes conventional feedback-control difficulty, especially when the corner frequency of the input filter is on top of the corner frequency of the output filter. With One-Cycle Control, the dynamics of the converter are made insensitive to the input filter; hence, the control-to-output transfer function is equivalent to a second order system of the output filter. Therefore, an output feedback loop can be easily implemented when it is necessary.

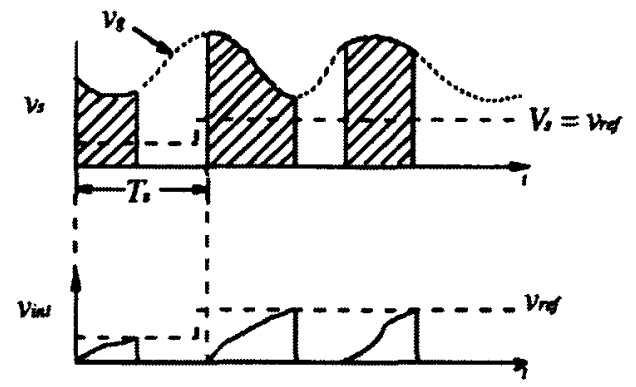

Fig. 4. Variable control reference.

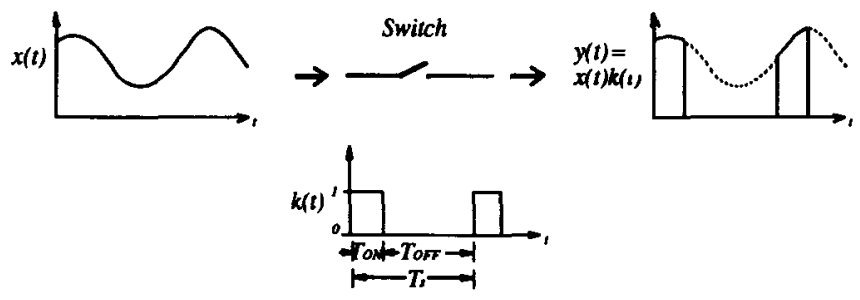

Fig. 5. The switch function.

\section{B. One-Cycle Control Theory}

A switch operates according to the switch function $k(t)$ at a frequency $f_{s}=1 / T_{s}$,

$$
k(t)= \begin{cases}1 & 0<t<T_{\mathrm{ON}} \\ 0 & T_{\mathrm{ON}}<t<T_{s} .\end{cases}
$$

In each cycle, the switch is on for a time duration $T_{\mathrm{ON}}$ and is off for a time duration $T_{\mathrm{OFF}}$, where $T_{\mathrm{ON}}+T_{\mathrm{OFF}}=T_{s}$. The duty-ratio $d=T_{\mathrm{ON}} / T_{s}$ is modulated by an analog control reference $v_{\text {ref }}(t)$. The input signal $x(t)$ at the input node of the switch is chopped by the switch and transferred to the output node of the switch to form a switched variable $y(t)$. The frequency and the pulse width of the switched variable $y(t)$ is the same as that of the switch function $k(t)$, while the envelope of the switched variable $y(t)$ is the same as the input signal $x(t)$, as shown in Fig. 5

$$
y(t)=k(t) x(t) .
$$

Suppose the switch frequency $f_{s}$ is much higher than the frequency bandwidth of either the input signal $x(t)$ or the control reference $v_{\text {ref }}(t)$; then the effective signal carried in the switch output, i.e. the average of the switched variable is

$$
\begin{aligned}
y(t) & =\frac{1}{T_{s}} \int_{0}^{T_{\mathrm{ON}}} x(t) d t \\
& \approx x(t) \frac{1}{T_{s}} \int_{0}^{T_{\mathrm{ON}}} d t \\
& =x(t) d(t) .
\end{aligned}
$$

The switched variable $y(t)$ at the output node of the switch is the product of the input signal $x(t)$ and the duty-ratio $d(t)$; therefore, the switch is nonlinear.

If the duty-ratio of the switch is modulated such that the integration of the switched variable at the switch output is 


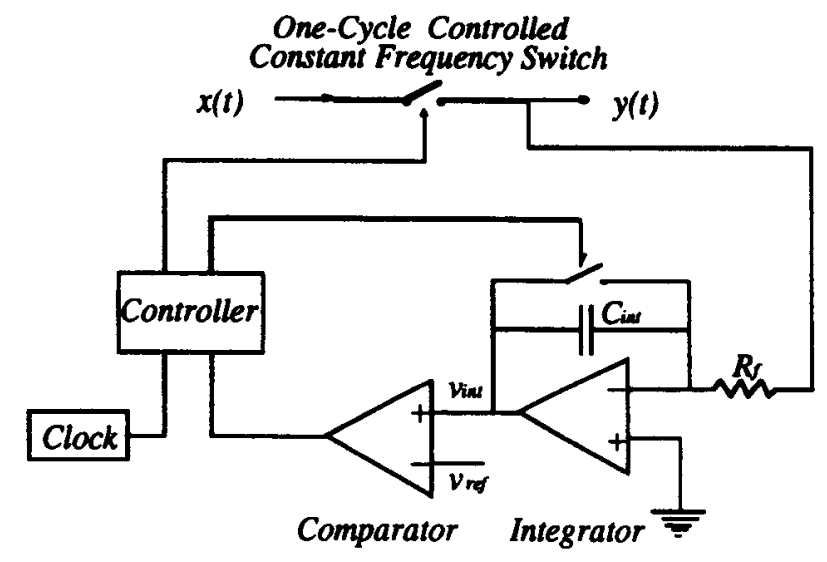

Fig. 6. The one-cycle controlled constant frequency switch.

exactly equal to the integration of the control reference in each cycle, i.e.

$$
\int_{0}^{T_{\mathrm{ON}}} x(t) d t=\int_{0}^{T_{s}} v_{\mathrm{ref}}(t) d t
$$

then the average value of the switched variable at the switch output is exactly equal to control reference in each cycle, since the switching period is constant. Therefore, the average of the switched variable is instantaneously controlled within one cycle, i.e.

$$
\begin{aligned}
y(t) & =\frac{1}{T_{s}} \int_{0}^{T_{\mathrm{ON}}} x(t) d t \\
& =\frac{1}{T_{s}} \int_{0}^{T_{s}} v_{\mathrm{ref}}(t) d t=v_{\mathrm{ref}}(t) .
\end{aligned}
$$

The technique to control switches according to this concept is defined as the One-Cycle Control technique. With One-Cycle Control, the effective output signal of the switch is

$$
y(t)=v_{\text {ref }}(t) \text {. }
$$

The switch fully rejects the input signal and linearly all-passes the control reference $v_{\text {ref }}$; therefore, the One-Cycle Control technique turns a non-linear switch into a linear path.

The implementation circuit for One-Cycle Control led constant-frequency switch is shown in Fig. 6. The key component of the One-Cycle Control technique is the integrator and the resetter. The integration starts the moment when the switch is turned on by the fixed frequency clock pulse. The integration value,

$$
v_{\text {int }}=k \int_{0}^{t} x(t) d t
$$

is compared with the control reference $v_{\text {ref }}(t)$ instantaneously, where $k$ is a constant. At the instant when the integration value $v_{\text {int }}$ reaches the control reference $v_{\text {ref }}(t)$, the controller sends a dommand to the switch to change it from the on state to the off state. At the same time, the controller resets the integrator. to zero. The duty-ratio $d=T_{\mathrm{ON}} / T_{s}$ of the present cycle is determined by the following equation:

$$
k \int_{0}^{d T_{s}} x(t) d t=v_{\text {ref }}(t)
$$

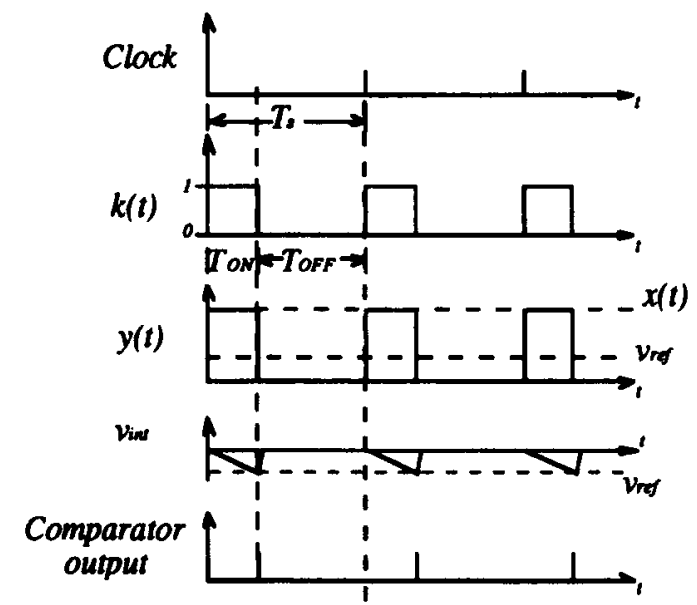

Fig. 7. The waveforms of the one-cycle controlled constant frequency switch.

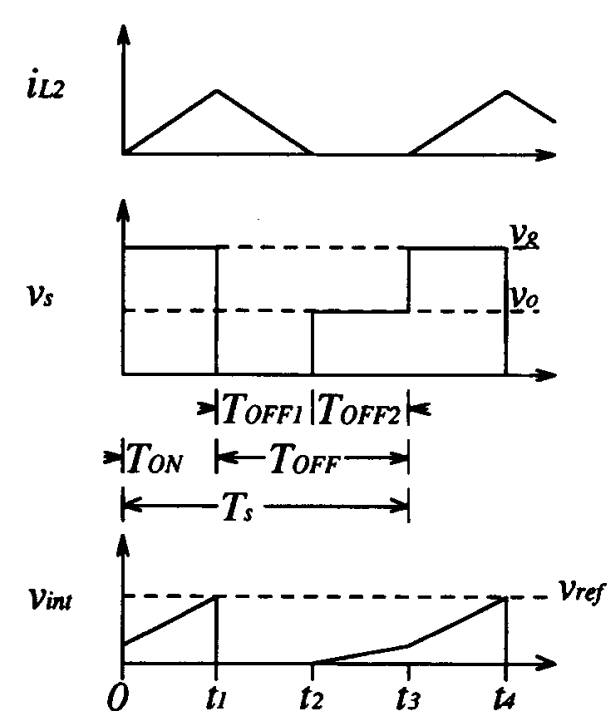

Fig. 8. One-cycle control of buck converter at discontinuous conducting condition.

Since the switch period $T_{s}$ is constant and $K=1 / k T_{s}$ is a constant, the average value of the switched variable at the switch output $y(t)$ is guaranteed to be

$$
y(t)=\frac{1}{T_{s}} \int_{0}^{d T_{s}} x(t) d t=K v_{\mathrm{ref}}(t)
$$

in each cycle. Fig. 7 shows the operating waveforms of the circuit, when $v_{\text {ref }}=$ constant.

Note that any physical or signal switch can be One-Cycle Controlled, i.e. the switched variable can be any switched physical variable or abstract signal.

\section{Discontinuous Conduction Mode}

One-Cycle Control is preserved when the converter operates under the condition of discontinuous conduction, provided the integrator reset time is smaller than the inductor discharge time. Take the buck converter shown in Fig. 1 as an example. The diode-voltage in the discontinuous mode is shown in Fig. 8. At $t=t_{1}$, the transistor is turned OFF, the inductor 


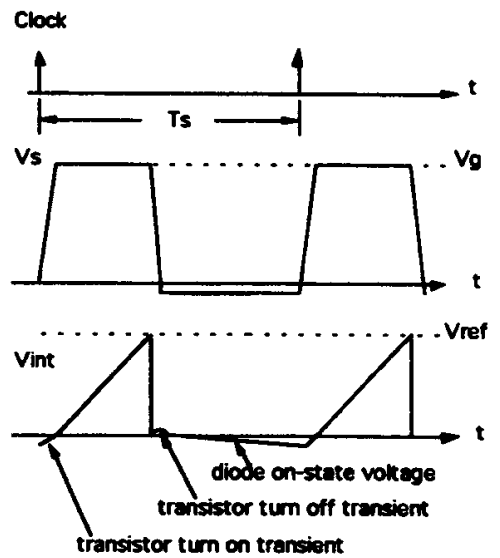

Fig. 9. Switching error correction.

current starts to decrease. During the time from $t_{1}$ to $t_{2}$, the diode conducts; therefore, the diode-voltage is $v_{s}=0$. At $t=$ $t_{2}$, the inductor runs out of current, while the transistor is still OFF. During the time from $t_{2}$ to $t_{3}$, the diode-voltage is equal to the output voltage. The integration starts immediately when the diode-voltage arises. At $t=t_{3}$, the switch is turned $\mathrm{ON}$ by the clock, the diode-voltage jumps to the power source voltage level, and the integration slope jumps up accordingly. When the integrated diode-voltage reaches the control reference, the switch is turned off.

$$
v_{\text {int }}=k\left(\int_{t_{2}}^{t_{3}} v_{o} d t+\int_{t_{3}}^{t_{4}} v_{g} d t\right)=v_{\text {ref }} .
$$

The output voltage $v_{0}$ equals the average value of the diodevoltage over the switch cycle; therefore, One-Cycle Control remains valid even if the switching converter operates in the discontinuous mode.

\section{Automatic Switching Error Correction}

In the above analysis, it is assumed that the switches of converters are ideal. In reality, switches will have finite switching time and finite on-state voltage. With One-Cycle Control, these switching errors are automatically corrected, providing the integrator reset time is substantially smaller than the switching time of the switch. Take the buck converter shown in Fig. 1 as an example, the transistor has finite turn-on and turn-off time and the diode has a voltage drop across it while it is conducting. The integrator integrates the transistor turn-off transient, the diode on-state voltage, the transistor turn-on transient, and the diode off-state voltage as shown in Fig. 9. The output of the integrator is compared with the control reference such that the controller turns off the transistor when the integrated value reaches the control reference. The average value of the diode voltage is equal to the control reference in each cycle regardless of the switching errors.

\section{E. Comparison with the ASDTIC Control}

One-Cycle Control is sometimes incorrectly confused with ASDTIC control [7], since both the One-Cycle Control method and the ASDTIC control method use an integrator. However, a more detailed comparison, outlined below, clearly exposes

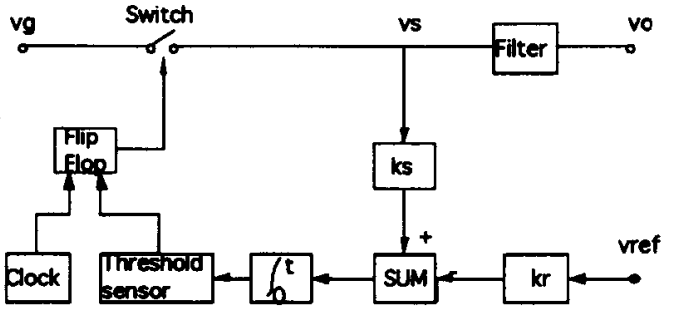

Fig. 10. Constant frequency ASDTIC control diagram.

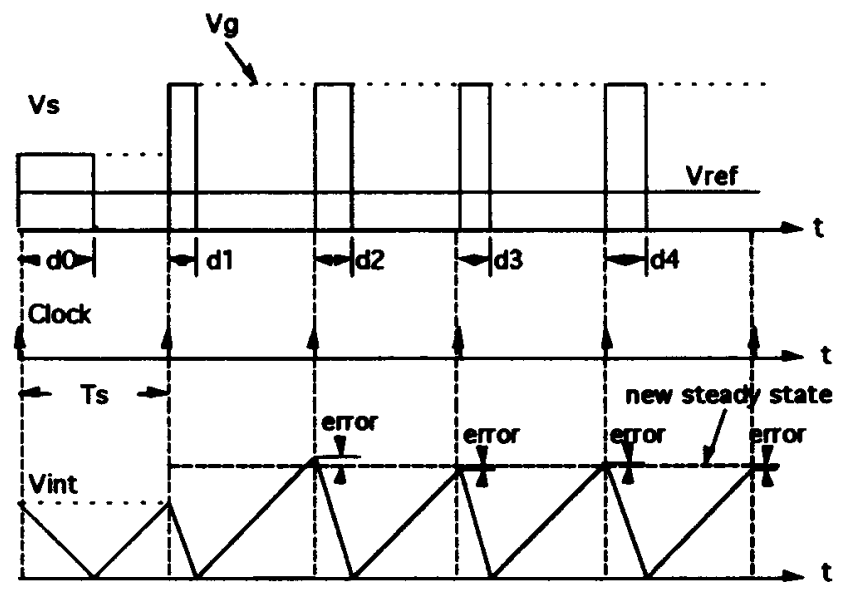

Fig. 11. The transient waveform of the ASDTIC control when $d<0.5$.

the fundamental differences between the two control methods and their different performance characteristics.

The constant frequency ASDTIC circuit [7] is shown in Fig. 10. The power source voltage $v_{g}$ is chopped by the switch to form the switched variable $v_{s}$. The clock signal sets the Flip/Flop, which subsequently turns on the switch. The summation of the control reference $-k_{r} v_{\text {ref }}$ and the switched variable $k_{s} v_{s}$ is integrated continuously. The output of the integrator is compared with a threshold signal and it produces a reset signal for the Flip/Flop when the integrated error during that switching cycle reaches zero, which subsequently turns off the switch. The duty-ratio of the switch in the present cycle is determined by some states and the duty-ratio of the previous cycle:

$$
d_{k}=\frac{v_{\text {ref }(k-1)}}{v_{g(k)}-v_{\operatorname{ref}(k)}}\left(1-d_{k-1}\right), \quad k=1,2,3 \cdots .
$$

This dependence on the history certainly ensures that a transient will last more than one switching cycle and may lead to instability.

For example, if the control reference is constant while the power source voltage $v_{g}$ is perturbed by a step function, the integration slope is immediately affected by the step function and so is the duty-ratio $d$. Suppose the duty-ratio before the step up transient is $d_{0}$, the duty-ratio during the step-up transient is $d_{k}$, where $k$ indicates the $k$ th cycle after the step up and $A=v_{\text {ref }} / v_{g}-v_{\text {ref }}$.

$$
d_{k}=\frac{A\left(1-(-A)^{k+1}\right)}{1+A}+(-A)^{k} d_{0}
$$




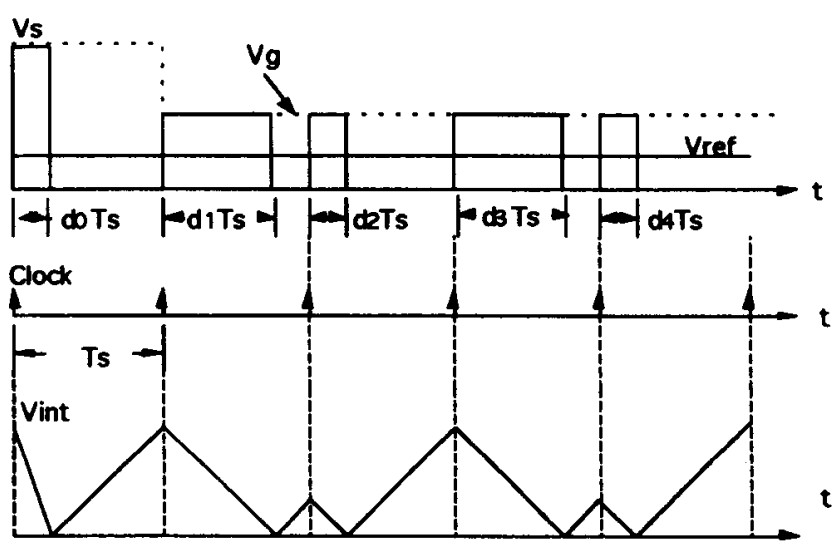

Fig. 12. The transient waveform of the ASDTIC control when $d=0.5$.

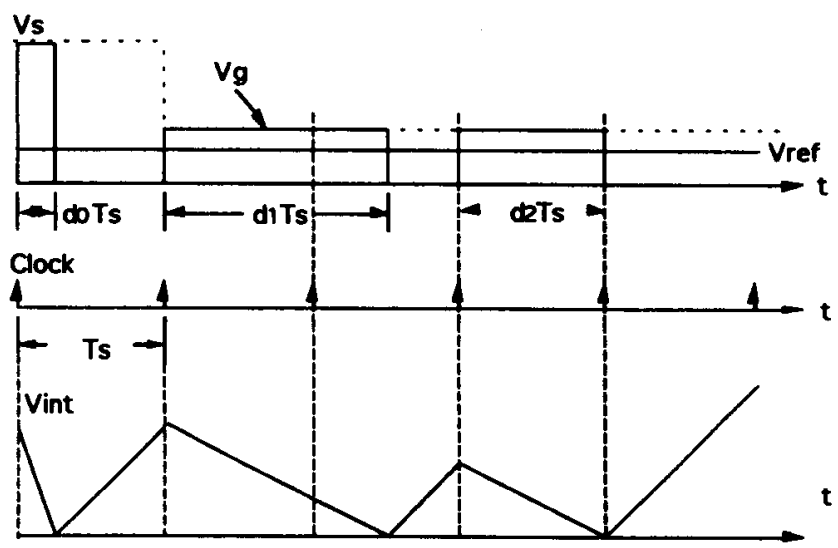

Fig. 13. The transient waveform of the ASDTIC control when $d>0.5$.

When $A=v_{\text {ref }} / v_{g}-v_{\text {ref }}$ is less than 1, i.e. the expected duty-ratio $d<0.5$, the transient converges to the new steadystate after many switching cycles as illustrated in Fig. 11. This control method is able to achieve zero output error in the steady-state, but only after many switching cycles. During a transient, this control method is unable to reach the new steady-state in one switching cycle. Therefore, it yields a zero steady-state error but a non-zero dynamic error between the control reference and the average value of the switched variable.

When $A=v_{\text {ref }} / v_{g}-v_{\text {ref }}$ is equal to 1 , i.e. the expected duty-ratio $d=0.5$, the transient does not converge; instead, it undergoes a subharmonic oscillation as shown in Fig. 12.

When $A=v_{\text {ref }} / v_{g}-v_{\text {ref }}$ is greater than 1, i.e. the expected duty-ratio $d>0.5$, the transient does not converge and the control circuit losses its control as shown in Fig. 13.

The subharmonic oscillation and the instability were previously recognized by the ASDTIC inventor himself, who imposed a limit on the allowable duty-ratios to avoid this problem [7].

In contrast, the duty-ratio of the switch in the One-Cycle Control method purely depends on the states of the current switching cycle, i.e.

$$
\frac{1}{T_{s}} \int_{0}^{d T_{s}} v_{s} d t=K v_{\text {ref }}
$$

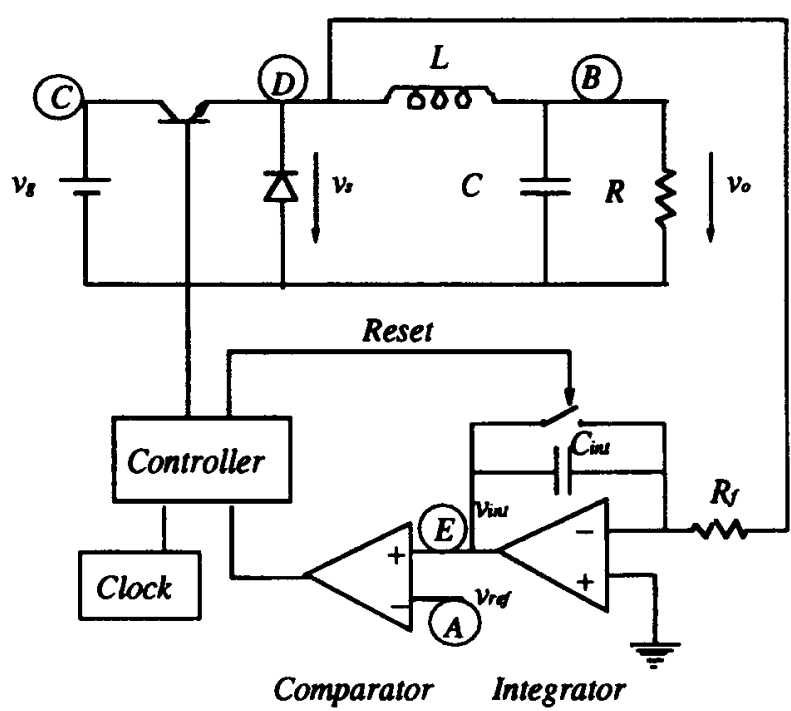

Fig. 14. One-cycle control of buck converter.

This history independence is the result of the resetter that clears the history in each cycle. Therefore One-Cycle Control led switch is robust in any of the above operating conditions. In addition, by employing One-Cycle Control, a transient of a switched variable lasts for only one switching cycle. There is no steady-state error nor dynamic error between the control reference and the average value of the switched variable.

\section{ONE-CYCLE CONTROL EXPERIMENTS}

Experiments were conducted to study the feasibility of OneCycle Control. The circuit used for the experiments is shown in Fig. 14. The operating condition for the experiments is $V_{g}=$ $15 \mathrm{~V}, f_{s}=30 \mathrm{kHz}, L=0.48 \mathrm{mH}, C=30 \mu \mathrm{F}, R=25 \Omega$. A, $\mathrm{B}, \mathrm{C}, \mathrm{D}$, and $\mathrm{E}$ are the test points used in the experiments.

A pulse from a constant frequency clock turns the transistor on and activates the integrator at the beginning of each switching cycle. The diode-voltage is fed back to an integrator. The integration value starts growing from zero and is compared with the control reference instantly. When the output voltage of the integrator reaches the control reference, the transistor is immediately turned off so that the average value of the switched variable in that cycle is exactly equal to or proportional to the control reference, and the integration is reset to zero so that the integration value is zero for the beginning of the next cycle.

In each cycle, the diode-voltage waveform may be different; however, as long as the area under the diode-voltage waveform in each cycle equals the control reference, instantaneous control of the switched variable, the diode-voltage $v_{s}$, is achieved.

\section{A. Rejection to Power Source Perturbation}

Suppose the control reference and the load are constant while the power source voltage $v_{g}$ is perturbed by an arbitrary pattern. The changing diode-voltage is integrated and the slope of the integrated diode-voltage changes immediately when 


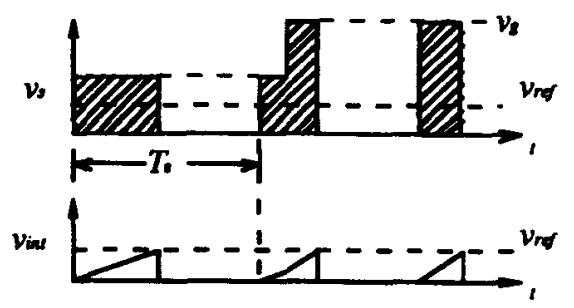

Fig. 15. Prediction of rejection to power source perturbations.

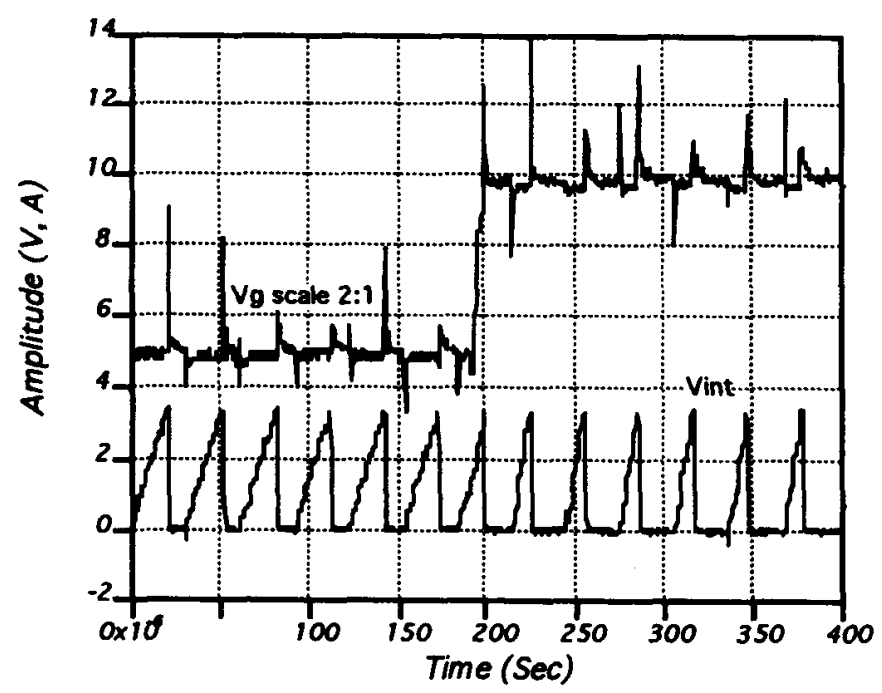

Fig. 16. Measurement of rejection to power source perturbations.

changes occur in the diode-voltage. Therefore, the power source directly and instantly affects the duty-ratio $d$ such that the integration of the diode-voltage is constant in each cycle.

In Fig. 15 the power source voltage is stepped up while the transistor is on. The slope of the integration changes immediately; therefore, the speed to reach the control reference is adjusted instantaneously in order to keep the integrated value of the diode-voltage equal to the control reference. It is predicted that this control technique completely rejects power source perturbations.

Experiment 1: The response of the diode-voltage to a stepup perturbation of the power source voltage was measured. A step-up function from $10-20 \mathrm{~V}$ was injected into the power source voltage $v_{g}$ at Point $\mathrm{C}$, while the load and the control reference were held constant. The response of the integrator $v_{\text {int }}$ was measured at Point E. Note that the power source voltage has been scaled down by a factor of two in order to fit on the plot shown in Fig. 16. The spikes on the power source voltage are caused by the nonzero impedance of the power source. These spikes did not influence the average value of the diode-voltage, because the spikes are included in the integration that is compared to the reference voltage. The power source voltage stepped up while the transistor was on and the slope of the integration of that cycle changed immediately; therefore, the duty-ratio was adjusted instantaneously.

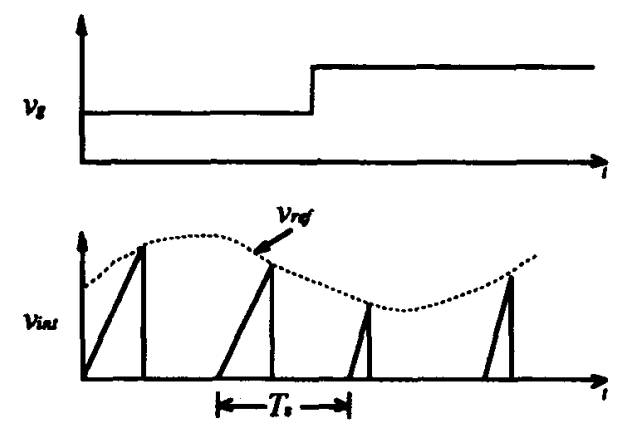

Fig. 17. Prediction of following the control reference and rejecting the power source perturbation.

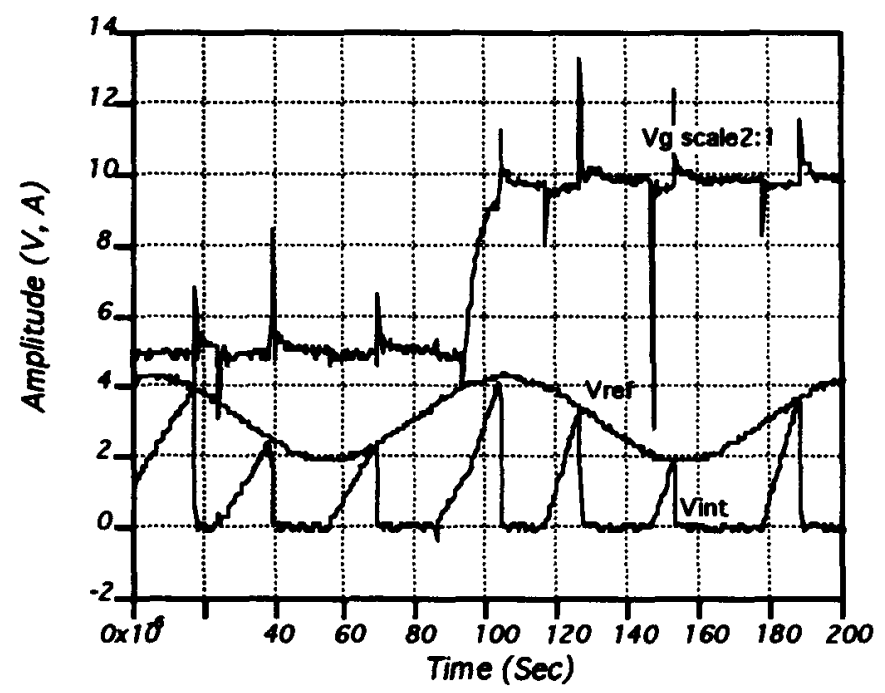

Fig. 18. Measurement of following the control reference and rejecting the power source perturbation.

\section{B. Following the Control Reference}

Suppose the power source voltage and the control reference are both time dependent. For example, the power source voltage has a step up perturbation while the control reference varies sinusoidaly. The slope of the integration becomes steeper when the amplitude of the power source voltage steps up. No matter how the integration slope changes, the integration value always keeps up with the sinusoidal control reference in each cycle. Therefore, the average value of the diode-voltage should not see the power source perturbation and it follows the control reference in one cycle, as predicted in Fig. 17.

Experiment 2: The capability of the diode-voltage to reject a step-up power source perturbation while following a sinusoidal varying control reference was measured. A step-up function from $10-20 \mathrm{~V}$ was injected into the power source voltage at Point $C$, while the control reference was varied with a sinusoid wave $v_{\text {ref }}=3.1+1.2 \sin \omega t, f=10 \mathrm{kHz}$, at Point A. The output response of the integrator was measured at Point E. Note that the power source voltage has been scaled down by a factor of two in order to fit on the plot shown in Fig. 18. The slope of the integration changed immediately when the power source voltage stepped up. The envelope of 


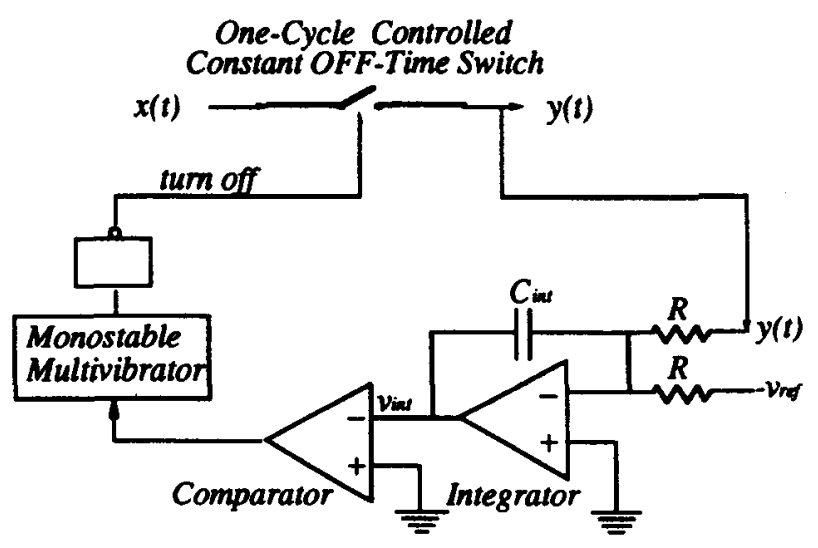

Fig. 19. The one-cycle controlled constant OFF-time switch.

the integration waveform kept up with the control reference exactly. Therefore, the average of the diode-voltage was not influenced by the power source disturbance and was fully controlled by the control reference.

\section{EXTENSION OF ONE-CYCLE CONTROL}

The One-Cycle Control technique conceived for the constant frequency switching converter is extended to control any type of switch. Four types of switches are considered here:

\begin{tabular}{|c|c|c|c|}
\hline Switch type & $T_{\mathrm{ON}}$ & $T_{\mathrm{OFF}}$ & $T_{s}$ \\
\hline constant frequency & variable & variable & constant \\
\hline constant ON-time & constant & variable & variable \\
\hline constant OFF-time & variable & constant & variable \\
\hline variable & variable & variable & variable \\
\hline
\end{tabular}

A constant OFF-time switch is used to describe the extension of this technique. The constant $\mathrm{ON}$-time and the variable switches can be implemented in a similar way.

For a constant OFF-time switch, $T_{\mathrm{OFF}}$ is constant. The object of One-Cycle Control is to adjust the ON-time $T_{\mathrm{ON}}$ in each cycle, such that the average value of the switched variable is exactly equal to the control reference.

The implementation circuit for One-Cycle Control of a constant OFF-time switch is shown in Fig. 19. The monostable multivibrator generates a constant pulse width, which is inverted by an inverter. The integration is started the moment the switch is turned off. From $t=0$ to $t=T_{\mathrm{OFF}}, v_{\text {int }}$ increases. When the monostable multivibrator changes its state from high to low, the switch is turned on. From $t=T_{\mathrm{OFF}}$ to $t=T_{s}, v_{\text {int }}$ decreases.

$$
v_{\mathrm{int}}= \begin{cases}\int_{0}^{t} v_{\mathrm{ref}}(t) d t & 0<t<T_{\mathrm{OFF}} \\ \int_{0}^{t} v_{\mathrm{ref}}(t) d t-\int_{T_{\mathrm{OFF}}}^{t} x(t) d t & T_{\mathrm{OFF}}<t<T_{s} .\end{cases}
$$

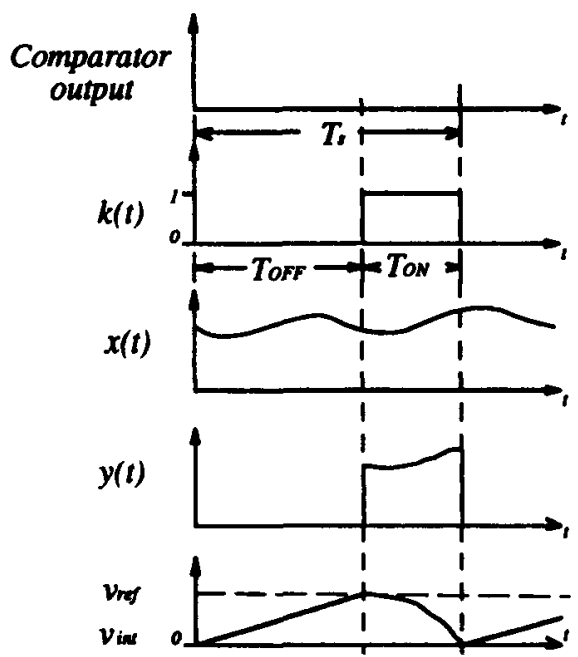

Fig. 20. The waveforms of the one-cycle controlled constant OFF-time switch.

At the instant when $v_{\text {int }}$ reaches zero, the comparator changes its state from low to high, which triggers the monostable multivibrator to high and turns the switch off. The present switching cycle is completed and the switch starts the next cycle.

The ON-time $T_{\mathrm{ON}}$ of the present cycle is determined by the following equation:

$$
\int_{T_{\mathrm{OFF}}}^{T_{\mathrm{OFF}}+T_{\mathrm{ON}}} x(t) d t=\left(T_{\mathrm{ON}}+T_{\mathrm{OFF}}\right) v_{\mathrm{ref}}(t)
$$

Since the OFF-time $T_{\mathrm{OFF}}$ of the switch is constant and the ON-time $T_{\mathrm{ON}}$ is controlled, the average value of the waveform at the switch output $y(t)$ is guaranteed to be

$$
y(t)=\frac{1}{T_{s}} \int_{T_{\mathrm{OFF}}}^{T_{\mathrm{OFF}}+T_{\mathrm{ON}}} x(t) d t=v_{\text {ref }}(t)
$$

in each cycle, where $T_{\mathrm{OFF}}$ is constant and $T_{s}$ is time variable. Fig. 20 shows the operating waveforms of the circuit.

The ASDTIC control [6] fits into constant ON-time switch category. The basic Delta modulation [17] fits into variable switching category.

Although the average value of the switched variable is equal to or proportional to the control reference in each switching cycle, the switching frequency can vary drastically under large signal dynamics. Therefore, in this extension, it is not justified to claim zero dynamic error, since a frequency modulation of the signal is present.

\section{CONCLUSION}

A large-signal nonlinear control technique, One-Cycle Control, is developed to dynamically control the duty-ratio $d$ of a switch, such that in each cycle the average of the switched variable at the switch output is exactly equal to or proportional to the control reference. Theoretically, converters with OneCycle Control are capable of rejecting the power source perturbations completely; the average value of the switched variable at the switch output node is able to follow the control reference within one cycle; and the switching error is 
automatically corrected within one cycle. There is no steadystate error nor dynamic error between the control reference and the average value of the switched variable.

When the One-Cycle Control technique is used to control a discrete switch, i.e. the input signal and the control reference of the switch are independent variables, One-Cycle Control is globally stable, since the duty-ratio of the switch in the One-Cycle Control method purely depends on the states of the current switching cycle. When a switch is embedded in a complex system such as the Cuk converter, the input signal of the switch may be a function of the output signal of the switch. In this case, a systematic method for global dynamic analysis and design using the Switching Flow-Graph method [15] is presented in [16].

The experiments with a One-Cycle Control led buck converter in this work yielded a very close match between the experimental measurements and the theoretical predictions.

The One-Cycle Control technique is very general and directly applicable to pulse-width-modulated, resonant-based, soft-switched switching converters, inverters, and rectifiers, for either voltage or current control in continuous or discontinuous conduction mode. Furthermore, it can be used to control any physical variable and abstract signal that is in the form of a switched variable or can be converted to the form of a switched variable.

The One-Cycle Control concept is straightforward and its implementation circuits are simple, yet it provides excellent control.

\section{ACKNOWLEDGMENT}

The authors acknowledge Prof. R. D. Middlebrook and Dr. Dragan Maksimović for many valuable discussions. Credit is due to Dr. Milivojo Brković and Dr. Narayana Sateesh Pillai for their assistance in the experimental work. The authors also wish to thank Dr. H. R. Visser and Dr. Andre Veltman for bringing the ASDTIC control papers to our attention at PESC 91.

\section{REFERENCES}

[1] K. M. Smedley, "Control art of switching converters," Ph.D. thesis, California Institute of Technology, Pasadena, 1990.

[2] K. M. Smedley and S. Ćuk, "One-cycle control of switching converters," in IEEE Power Electronics Specialists Conference, 1991 Record, pp. 1173-1180, (IEEE Publication 91ch3163-3). See also U.S. Patent $5,278,490$.

[3] A. Capel, G. Ferrante, D. O'Sullivan, and A. Weinberg, "Application of the injected current model for the dynamic analysis of the switching regulators with the new concept of $L C^{3}$ modulator," in IEEE Power Electronics Specialists Conference, 1978 Record, pp. 135-147 (IEEE Publication 78ch1337-5 AES)

[4] C. W. Deisch, "Simple switching control method changes power converter into a current source," in IEEE Power Electronics Specialists Conference, 1978 Record, pp. 300-306 (IEEE Publication 78ch1337-5 AES).

[5] R. D. Middlebrook and S. Cuk, "Advances in switched mode power conversion vols. I, II, \& III," TESLAco 1981 and 1983.

[6] F. C. Schwarz, "Analog signal to discrete time interval converter (ASDTIC)," U.S. Patent 3659184, 1972.

[7] _ "Engineering information on an analog signal to discrete time interval converter (ASDTIC)," NASA CR-134544, 1974.

[8] R. Venkataramanan, A. Sabanovic, and S. Cuk, "Sliding mode control of DC-to-DC converters," in Proc. IECON '85. 1985 Int. Conf. Industrial
Electronics, Control and Instrumentation (Cat. No. 85CH2160-0), vol. 1 , pp. 251-258.

[9] K. M. Smedley, "Fixed frequency sliding-mode control," Technique Notes, Power Electronics Group, California Institute of Technology, Pasadena, 1988.

[10] W. Tang, F. C. Lee, R. B. Ridley, and I. Cohen, "Charge control: Modeling, analysis and design," in IEEE Power Electronics Specialists Conference, 1992 Record, pp. 503-511 (IEEE Publication 92ch3163-3)).

[11] L. Malesani, L. Rossetto, G. Spiazzi, and P. Tenti, "Three-phase power factor controller with minimum output voltage distortion," in Proc. Intelec 93: I5th Int. Telecommunications Energy Conf., (Cat. No. 93CH3411-6), Paris, France, 27-30, vol. 2, pp. 463-468, Sept. 1993.

[12] E. Santi and S. Cuk, "Modeling of one-cycle controlled switching converters," in Fourteenth Int. Telecommunications Energy Conf. INTELEC '92, (Cat. No. 92CH3195-5). Washington, DC, Oct. 4-8, 1992, pp. 131-138.

[13] D. Maksimovic, Y. Jang, and R. Erickson, "Nonlinear-carrier control for high power factor boost rectifiers," in Proc. 1994 IEEE Applied Power Electronics Conf. and Exposition-ASPEC'95, Dallas, TX, vol. 2, pp. 635-641, Feb. 5-9, 1995.

[14] Z. Lai and K. Smedley, "A new extension of one-cycle control and its application to switching power amplifiers," in Proc. 1994 IEEE Applied Power Electronics Conf. and Exposition-ASPEC'95, Dallas, TX, vol. 2, pp. 826-831, Feb. 5-9, 1995.

[15] K. M. Smedley and S. Ćuk, "Switching flow-graph nonlinear modeling technique," IEEE Trans. Power Electron., vol. 9, no. 4, pp. 405-413, July 1994.

[16] "Dynamics of one-cycle controlled Cuk converter," this issue, pp. 634-640.

[17] P. Ziogas, "The delta modulation technique in static pwm inverter," IEEE Trans. Ind. Applicat., pp. 199-204, Mar., 1981.

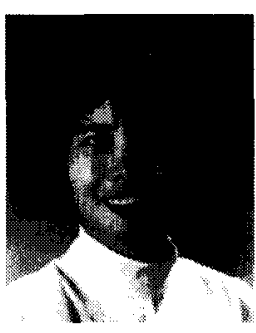

Keyue M. Smedley (M'87) received the B.S. and M.S. degrees in electrical engineering from Zhejiang University, China, in 1982 and 1985 , respectively, and the Ph.D. degree in electrical engineering from the California Institute of Technology, Pasadena, in 1991.

She worked as an engineer at the Superconducting Super Collider from 1990 to 1992, where she was responsible for determining the electrical characteristics of the superconducting magnet strings and designing the power conversion system for the Superconducting Collider. She has been a faculty member at the University of California, Irvine, since 1992. She established a state-of-theart Power Electronics Laboratory at UCI. Her areas of interest include nonlinear modeling and control of switching converters, switching circuit topologies, switching power amplifications, power factor corrections, and electrical characteristics of superconducting magnets. She currently holds a U.S. patent for One-Cycle Control.

Dr. Smedley is a faculty member of Eta Kappa Nu.

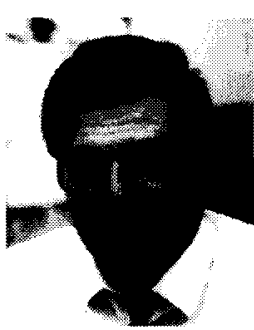

Slobodan Ćuk (M'77-SM'95) is a full-time Professor of Electrical Engineering at the California Institute of Technology, Pasadena.

He founded TESLAco in 1980, with the charter to apply the basic research results developed at Caltech to practical commercial and military designs. He conducts research in electrical energy processing systems, and teaches courses in Power Electronics and Fundamentals of Energy Processing

For the invention of a new switching converter topology, now known as the ĆUKonverter ${ }^{(B)}$, and several of its extensions, Dr. Ćuk won a coveted IR*100 Award for 1980 from Industrial Research Magazine. For the invention of Integrated Magnetics and expediting the development of Switched-Mode power converters, he obtained a prestigious 1991 Edward Longstreth Medal from the Franklin Institute. His publications include over 100 scientific papers in the power electronics field and a three volume book on Switched-Mode power conversion. 